\title{
Palatal mucormycosis in neutropenic children: A Case Report with Review of Literature
}

\author{
K. Devaraja ${ }^{1}$ Neethu V. Krishnan ${ }^{1}$ Vasudeva K. Bhat ${ }^{2} \quad$ Kailesh Pujary $^{1}$ Archana M. Venkatagiri ${ }^{2}$ \\ Kalasekhar Vijayasekharan ${ }^{2, *(1)}$ \\ ${ }^{1}$ Department of Otorhinolaryngology and Head and Neck Surgery, \\ Kasturba Medical College, Manipal, Manipal Academy of Higher \\ Education, Manipal, Karnataka, India \\ ${ }^{2}$ Division of Pediatric Hematology and Oncology, Manipal Comprehensive

\begin{abstract}
Address for correspondence K. Devaraja, MS, Department of Otorhinolaryngology and Head and Neck Surgery, Kasturba Medical College, Manipal, Manipal Academy of Higher Education, Manipal, Udupi, Karnataka, 576104, India (e-mail: deardrdr@gmail.com).
\end{abstract} Cancer Care Centre, Kasturba Medical College, Manipal, Manipal Academy of Higher Education, Manipal, Karnataka, India

Ind J Med Paediatr Oncol 2021;42:491-495.

\begin{abstract}
Keywords

- febrile neutropenia

- invasive fungal disease

- palatal mucormycosis

- mucormycosis

- amphotericin-B

- posaconazole

Palatal involvement in mucormycosis is mostly secondary to rhino-orbito-cerebral disease, but rarely can be a primary disease of the oral mucosa. This report presents two rare cases of the isolated palatal mucormycosis in neutropenic children and highlights some of the peculiar features of the primary palatal disease and management-related issues in children. A 12-year-old child, who had completed the dexamethasone-based induction phase of chemotherapy for Near Early $T$ cell precursor acute lymphoblastic leukemia, and a 9-yearold boy with a Late Isolated Medullary relapse of B cell acute lymphoblastic leukemia, who was to receive salvage induction chemotherapy, developed palatal discoloration without any other major complaints. Both had neutropenia and were on antifungal prophylaxis. In vitro staining of the discolored mucosa suggested mucormycosis, which was confirmed by pathological examination of the debrided tissue. Computed tomography, done before debridement, showed no significant sinonasal disease enabling us to proceed with the transoral approach. With the help of adjuvant antifungal therapy, the infection could be contained in both cases. This report, along with the reviewed literature, shows that limited palatal mucormycosis can be effectively treated by early diagnosis and debridement and appropriate antifungal therapy. Also, the role of antifungal prophylaxis amongst neutropenic patients has been briefly discussed here.
\end{abstract}

\section{Introduction}

Mucormycosis is an emerging global disease with high morbidity and mortality. It is caused by saprophytic fungi belonging to Rhizopus and Mucor species and is mostly associated with immunocompromised conditions such as diabetes mel-

\footnotetext{
Present address: Department of Pediatric Oncology, Regional Cancer Center, Trivandrum, Kerala, India
}

DOI https://doi.org/ 10.1055/s-0041-1739188. ISSN 0971-5851. litus or neutropenia secondary to chemotherapy. ${ }^{1-3}$ The etiopathology of this invasive fungal infection is attributed to the inhalation of fungal sporangiospores or the direct inoculation of organisms through the disrupted surface of skin or mucosa in vulnerable patients. ${ }^{1}$ The majority of these cases manifest as rhino-orbito-cerebral mucormycosis, but can also present as cutaneous, pulmonary, gastrointestinal, and disseminated diseases. ${ }^{1-3}$ However, mucormycosis involving only the hard palate or the alveolar process of the maxilla or mandible is
(C) 2021. Indian Society of Medical and Paediatric Oncology. All rights reserved.

This is an open access article published by Thieme under the terms of the Creative Commons Attribution-NonDerivative-NonCommercial-License, permitting copying and reproduction so long as the original work is given appropriate credit. Contents may not be used for commercial purposes, or adapted, remixed, transformed or built upon. (https://creativecommons.org/ licenses/by-nc-nd/4.0/)

Thieme Medical and Scientific Publishers Pvt. Ltd., A-12, 2nd Floor, Sector 2, Noida-201301 UP, India 
reported very rarely. ${ }^{4-6}$ Also, there are only a handful of reports in the literature about mucormycosis among children. $^{7-10}$ This case report presents the clinical courses of two rare cases of palatal mucormycosis, seen in children who were on acute lymphoblastic leukemia (ALL) therapy, along with an appropriate literature review.

\section{Case 1}

A 12-year-old child, who had completed the dexamethasonebased induction phase of chemotherapy for Near Early T cell precursor acute lymphoblastic leukemia (ETP-ALL), was admitted to the high dependency unit of pediatric hematooncology with febrile neutropenia. The child was on fluconazole for Candida tropicalis septicemia when he developed a blackish crust on the left side upper alveolus and adjacent hard palate. While the otorhinolaryngology team was called for further evaluation of this new symptom, the patient was started on conventional amphotericin B with the starting dose of $0.5 \mathrm{mg} / \mathrm{kg} /$ day, which was increased to $1.5 \mathrm{mg} / \mathrm{kg} /$ day after 2 days. On enquiring further, there were no associated complaints of nasal discharge, blockage, postnasal drip, or any ear or throat complaints. General examination revealed that the boy was febrile and had tachycardia. On local examination, there was a black eschar of $3 \mathrm{~cm} \times 2 \mathrm{~cm}$ along the left maxillary alveolus extending to the adjacent hard palate, as shown in -Fig. 1A. The lesion had involved the canine and the first two left maxillary premolars, with a visible oroantral fistula along the labial surface of the alveolus. Potassium hydroxide $(\mathrm{KOH})$ mount from the scrapings of the lesion revealed filamentous fungal elements in the form of broad, sparsely septate hyphae, branching at 90 degrees, suggestive of mucormycosis. Blood counts showed severe neutropenia with thrombocytopenia. Computed tomography of the paranasal sinuses, depicted in - Fig. 1B, showed mucosal thickening in the ipsilateral maxillary sinus, without any bone erosion. The patient was taken up for emergency surgical debridement via a transoral approach, with adequate platelet transfusions and reserve. Intraoperatively, the debridement of dead bone accompanied the removal of the left upper canine, premolar, and first molar for disease clearance. On-table examination of the mucosa of the left maxillary sinus was found to be not involved except on the floor, adjacent to the diseased alveolus. Following the surgical debridement, the patient continued to receive conventional amphotericin $B$ for 28 days, during which he developed intermittent but marked electrolyte disturbance, requiring daily potassium and magnesium corrections. He was also started on interim 6 mercaptopurine within 48 hours of surgery, for a duration of 14 days, following which the consolidation chemotherapy was continued. The last week of therapy with amphotericin B was overlapped with tablet posaconazole, at the dose of $300 \mathrm{mg}$ twice daily on the first day and $300 \mathrm{mg}$ once a day thereafter. The patient was discharged on tablet posaconazole, which he took until the end of the intensive phase of chemotherapy that lasted nearly 5 months. As demonstrated in - Fig. 1C, the palatal wound healed well without any evidence of recurrence on three subsequent follow-up visits 1 month apart. The nasogastric tube was removed in the second month of surgery, and the oral feeds were started successfully without nasal regurgitation. Currently, the patient is in remission and free of fungal disease, and awaiting prosthodontic rehabilitation for missing teeth.

\section{Case 2}

A 9-year-old boy with a Late Isolated Medullary relapse of B cell ALL, admitted to pediatric oncology ward for salvage induction chemotherapy under voriconazole prophylaxis, complained of discoloration of mucosa over the hard palate. On examination, a $3 \mathrm{~cm} \times 3 \mathrm{~cm}$ black eschar was noted on the left side of the hard palate, adjacent to the alveolus from the upper lateral incisor until the second premolar posteriorly. The patient had no symptoms of pain or fever, nor he complained of any nasal or ear problems. With the clinical suspicion of breakthrough mucormycosis, otorhinolaryngology opinion was sought for emergency debridement. Meanwhile, computed tomography of the face demonstrated minimal mucosal thickening of the maxillary sinus on the same side, and the $\mathrm{KOH}$ mount from the mucosal crust showed fungal elements suggestive of mucormycosis. The patient was started on intravenous infusion of conventional amphotericin $B$ at the rate of $0.5 \mathrm{mg} / \mathrm{kg} /$ day and was taken up for debridement on the same day. A surgery similar to the first case was carried out under general anesthesia. The affected portion of alveolar process of the left maxilla was removed, from the left upper incisors until the first molar, and the entire specimen was sent for fungal culture and histopathology. On examining the maxillary sinus through the defect, it was found to be uninvolved in this case too. The

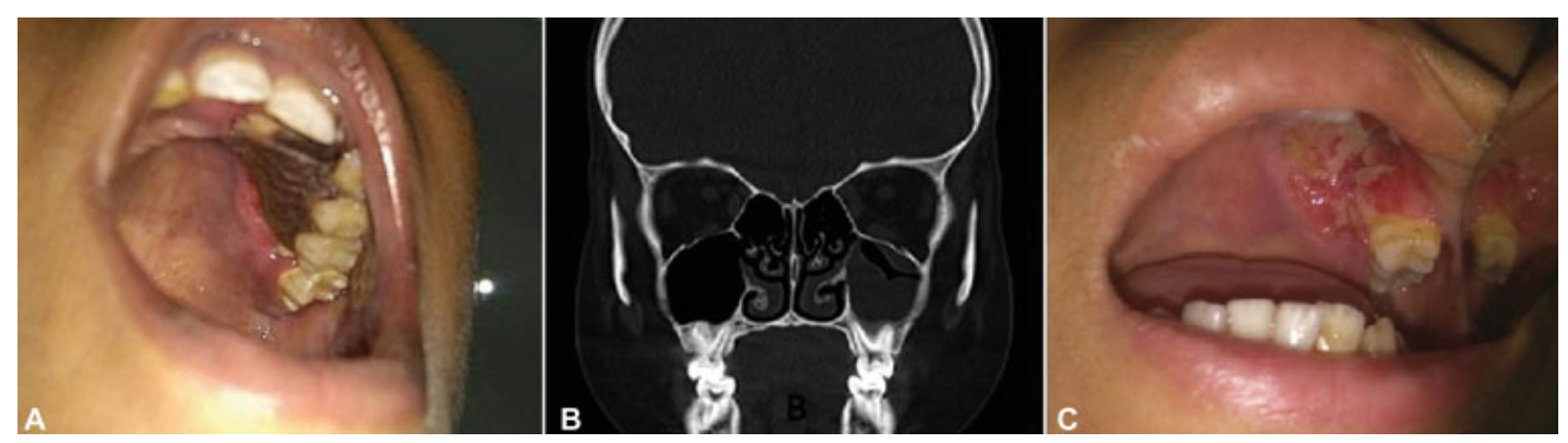

Fig. 1 Clinico-radiological images of case-1, showing necrosis of left hard palate adjacent to premolar teeth in A, the coronal image of computed tomography with minimal mucosal thickening in B, and healed surgical defect after a month of debridement in $\mathbf{C}$. 
patient had an uneventful postoperative recovery and continued receiving aggressive antifungal therapy in the form of conventional amphotericin B, $1.5 \mathrm{mg} / \mathrm{kg} / \mathrm{day}$, along with daily monitoring of electrolytes and blood counts. Postoperatively, he was started on oral chemotherapy, interim 6 mercaptopurine within 48 hours of surgery, which was followed up by maintenance chemotherapy per protocol. His histopathology from the excised specimen was reported as invasive mucormycosis and had also suggested concomitant cytomegalovirus (CMV) infection, which was confirmed subsequently by the elevated blood CMV DNA copy numbers. The surgical wound in the oral cavity was cleaned and monitored regularly for the possible progression of the disease. The patient also received ganciclovir for 14 days after the confirmation of CMV infection. Although the plan was to continue amphotericin for 28 days, overlapped with oral posaconazole during the last week, it had to be stopped after 21 days due to the severe and nonresponding electrolyte disturbance. The patient was started on syrup posaconazole at the dose of $5 \mathrm{~mL}$ thrice with a fatty meal. One month post-surgery, there was no evidence of recurrence in the oral or nasal cavity, with optimal healing of the surgical defect in the palate. Subsequently, the nasogastric tube was removed, and the child was started on oral feeds. The child was discharged on oral posaconazole, but he contracted novel Coronavirus-19 infection while on oral maintenance therapy after 2 weeks of discharge and succumbed to the same.

\section{Discussion}

Generally, palatal involvement in mucormycosis occurs secondary to the spread of invasive infection from sinonasal disease. ${ }^{11-14}$ Only rarely does mucormycosis occur inside the oral cavity, with isolated necrosis of the hard palate or alveolar process of maxilla or mandible..$^{3-5,8-10}$ Similarly, the occurrence of rhino-orbito-cerebral mucormycosis itself is not uncommon among neutropenic adult patients who are on chemotherapy for hematological malignancies, but the limited disease of the hard palate has been reported very rarely in neutropenic patients, that too in children. ${ }^{14-19}$ - Table 1 summarizes the published reports of mucormycosis limited to the oral palate, including those cases that involved children

Table 1 Previously published cases of palatal mucormycosis (without significant sinonasal disease)

\begin{tabular}{|c|c|c|c|c|}
\hline Author(s) and year & Demographics & Co-morbidity & Treatment & Outcomes \\
\hline Samanta et al, $2009^{9}$ & 8 year/boy & $\overline{A L L}$ & $\begin{array}{l}\text { Surgical debridement and } \mathrm{AMB}^{\mathrm{a}} \\
\text { (duration not known) }\end{array}$ & Recovered $^{\mathrm{b}}$ \\
\hline Abuali et al, $2009^{19}$ & 14 year/female & AML & $\begin{array}{l}\text { Posaconazole, caspofungin and } \\
\mathrm{AMB}^{\mathrm{C}}\end{array}$ & Did not recover \\
\hline \multirow[t]{2}{*}{ Al Akhrass et al, $2011^{20}$} & 31 year/male & AML & $\begin{array}{l}\text { Repeated surgical debridement, } \\
\text { voriconazole, AMB, and } \\
\text { posaconazole }^{c}\end{array}$ & Did not recover \\
\hline & 38 year/male & AML & $\mathrm{AMB}^{\mathrm{C}}$ & Did not recover \\
\hline Manjunatha et al, $2012^{24}$ & 50 year/male & $\mathrm{DM}$ & Surgical debridement and $\mathrm{AMB}^{\mathrm{a}}$ & Recovered \\
\hline Srivastava et al, $2015^{8}$ & 2 month/boy & $\mathrm{Nil}$ & $\begin{array}{l}\text { Surgical debridement and Vorico- } \\
\text { nazole for } 42 \text { days }\end{array}$ & Recovered \\
\hline Kalaskar et al, $2016^{10}$ & 18 month/boy & $\mathrm{Nil}$ & AMB for 31days & Recovered \\
\hline Rai et al, $2018^{4}$ & 57 year/male & DM & $\begin{array}{l}\text { Repeated surgical debridement and } \\
\text { topical clotrimazole and ketocona- } \\
\text { zole for } 7 \text { weeks }\end{array}$ & Partial recovery ${ }^{\mathrm{d}}$ \\
\hline France et al, $2018^{21}$ & 20 year/male & $\overline{A L L}$ & Posaconazole $^{c}$ & Did not recover \\
\hline Vučićević Boras et al, $2019^{23}$ & 54 year/male & $\overline{A L L}$ & $\begin{array}{l}\text { Surgical debridement, Posacona- } \\
\text { zole, and AMB for } 28 \text { days }\end{array}$ & Recovered \\
\hline De Oliveria et al, $2020^{16}$ & 92 year/male & Anemia & $\mathrm{AMB}^{\mathrm{C}}$ & Did not recover \\
\hline \multirow[t]{2}{*}{ Our study } & 9 year/boy & ALL & $\begin{array}{l}\text { Surgical debridement, AMB for } \\
28 \text { days and posaconazole for } 5 \\
\text { months }\end{array}$ & Recovered \\
\hline & 12 year/boy & ALL & $\begin{array}{l}\text { Surgical debridement, AMB for } \\
21 \text { days, and posaconazole for } 2 \\
\text { weeks }\end{array}$ & Recovered $^{\mathrm{e}}$ \\
\hline
\end{tabular}

Abbreviations: AMB: amphotericin B; AML, acute myeloblastic leukemia; ALL, acute lymphoblastic leukemia, DM, diabetes mellitus.

Note that prior to our report, there were only 3 case reports of palatal mucormycosis among children (colored rows).

${ }^{\mathrm{a}}$ The duration of AMB was not mentioned.

${ }^{b}$ Recovered from that episode of mucormycosis without any evidence of relapse even after discontinuing antifungal therapy.

cDied in hospital due to persistent disease.

${ }^{\mathrm{d}}$ Lost to follow-up during ongoing treatment but had shown significant clinical improvement to therapy.

${ }^{\mathrm{e}}$ Recovered from fungal disease but died due to novel Coronavirus-19 infection. 
with secondary neutropenia due to chemotherapy for hematological diseases. The two cases narrated here not only represent the rare form of mucormycosis but also involve an unusual age group, making this a peculiar case report. The following paragraphs discuss some of the salient features of isolated palatal mucormycosis, including the nuances related to its management in children and the controversy surrounding the voriconazole prophylaxis in neutropenic patients.

Contrary to the classical clinical manifestations of rhinoorbital mucormycosis that aid the diagnosis in most of those cases, the clinical diagnosis may not be so straightforward in cases of limited palatal mucormycosis. The presence of mucosal ulceration or necrosis on the palate without any sinonasal manifestations in the latter could rather mimic a malignancy or osteomyelitis., ${ }^{4-21}$ However, unlike in the case of neoplasm or osteomyelitis, the onset of palatal lesion is quite dramatic, and the progression is relatively rapid in invasive fungal disease. Despite the drastic clinical evolution of mucormycosis, the actual ulceration or necrosis of the hard palate and overt oroantral fistula are generally preceded by a slight discoloration of the mucosa or by a submucosal swelling. ${ }^{16}$ Such preceding clinical signs have also been reported in cases of rhino-orbital mucormycosis with palatal involvement. ${ }^{13,20}$ In other words, the regular monitoring of oral cavity mucosa by the clinicians or by oneself, or by parents in the case of children can aid in the early diagnosis of palatal mucormycosis in vulnerable patients. ${ }^{9,10}$ As such, the oral mucosa is prone to many inflammatory conditions and opportunistic infection in neutropenic children and would require regular surveillance. ${ }^{22}$ The treating team should have a high index of suspicion, and any doubtful lesion of the palatal mucosa in an immunocompromised patient is to be biopsied at the earliest to rule out the possibility of mucormycosis.

As per the recently published "global guideline for the management of mucormycosis," surgical debridement of the involved tissue remains a strongly recommended first-line therapy along with high-dose liposomal amphotericin $\mathrm{B}$, for almost all cases of mucormycosis, irrespective of the age of the patient and medical comorbidities. ${ }^{23}$ As it can be noticed in - Table 1, the chances of recovery seem to reduce if active surgical debridement is not considered early in the course. Some of the reasons for not considering the surgical debridement in previous reports were poor general condition, low platelet count, and most importantly, the fear of post-surgical morbidity in the form of oro-antral fistula. ${ }^{12,15-17}$ We proceeded with surgical debridement despite the low platelet count and possible postoperative morbidity, with adequate platelet cover and appropriate consent. Although the surgical intervention for palatal mucormycosis could pose a greater challenge in younger children with respect to restoring oral feeding, it needs to be done in confirmed cases of invasive fungal disease to prevent further disease progression and corresponding poorer outcome. Besides, there are several reports of successful rehabilitation of larger palatal defects even among infants as young as 2 to 3 months, supporting the decision to proceed with surgical debridement in younger children, whenever indicated. ${ }^{8-10}$ Even in our index cases, we could restore the oral feeding after the local site healed well. Because the size of the defect was not large and the affected children were relatively older, both of our patients could learn to swallow well without having the need for palatal prosthesis.

Apart from the timely surgical debridement and adequate antifungal therapy, the nature of the underlying co-morbidity and the area of involvement by the invasive fungal disease also seem to have prognostic role in mucormycosis cases. ${ }^{24,25}$ As per an analysis, mucormycosis in neutropenic patients tend to have poorer survival compared with similar illness in diabetic patients. ${ }^{25}$ However, most of the previous reports of mucormycosis in neutropenic children have exhibited a favorable outcome, attributing the therapeutic success to early diagnosis, adequate surgical debridement, and appropriate antifungal therapy. ${ }^{7-10,14}$ With respect to the area of disease involvement, a limited disease, localized only to the hard palate, probably contributes to the successful outcome seen in cases of palatal mucormycosis. ${ }^{8-10,18}$

Generally, neutropenic patients on intense chemotherapy tend to receive antifungal prophylaxis for the prevention of invasive fungal disease due to opportunistic fungi. Because invasive aspergillosis is common during chemotherapy-induced prolonged neutropenia, as opposed to mucormycosis in diabetes mellitus, voriconazole is the preferred antifungal agent of choice for prophylaxis in such neutropenic patients, and posaconazole is reserved for the latter. Interestingly, several reports have linked voriconazole prophylaxis in neutropenia to the occurrence of breakthrough mucormycosis. ${ }^{22,26,27}$ As per Kontoyiannis et al, $48 \%$ of their mucormycosis cases were on voriconazole prophylaxis. $^{27}$ However, there is an obvious skepticism about this association between voriconazole prophylaxis and breakthrough mucormycosis. ${ }^{28,29}$ A subsequent epidemiological study that compared the cumulative incidences of invasive mucormycosis before and after the commercial use of voriconazole downplayed the role of voriconazole prophylaxis in mucormycosis. ${ }^{30}$ This study attributed the increased number of mucormycosis cases after the introduction of voriconazole prophylaxis to an increase in the number of susceptible individuals. Lastly, although posaconazole can be a reliable alternative for prophylaxis in neutropenic patients, with the added benefit of preventing mucormycosis, the financial burden associated with longterm use of posaconazole is a major hurdle for its routine/universal clinical application, especially in developing nations. Nevertheless, apart from being used as an oral antifungal for treating the proven cases of mucormycosis, posaconazole should be used prophylactically during a subsequent episode of relapse of neutropenia in such cases, to prevent the reemergence of mucormycosis.

\section{Conclusion}

Mucormycosis can rarely involve only the alveolar process and adjacent palate. Although it is extremely rare in children with neutropenia, it can be managed successfully by timely surgical debridement and adequate antifungal therapy. 


\section{Authors' Contributions}

The manuscript has been read and approved by all authors. All authors have contributed to this manuscript. Specifically, Dr. K Devaraja and Dr. Neethu V Krishnan were involved in all stages of the study including conceptualization, study design, the definition of intellectual content, literature review, clinical studies, data acquisition and analysis, and manuscript preparation, editing, and reviewing. Dr. Vasudev K. Bhat and Dr. Kalasekhar were involved in conceptualization, the definition of intellectual content, literature review, clinical studies, and manuscript preparation, editing, and reviewing. Dr. Kailesh Pujary and Dr. Archana M V were also involved in conceptualization, study design, literature review, clinical studies, and manuscript editing and reviewing.

Funding

None.

\section{Conflict of Interest}

None declared.

\section{Declaration of Patient Consent}

The authors certify that they have obtained all appropriate patient consent forms.

\section{References}

1 Skiada A, Lass-Floerl C, Klimko N, Ibrahim A, Roilides E, Petrikkos $\mathrm{G}$. Challenges in the diagnosis and treatment of mucormycosis. Med Mycol 2018;56(Suppl 1):93-101

2 Serris A, Danion F, Lanternier F. Disease entities in mucormycosis. J Fungi (Basel) 2019;5(01):23

3 Bonifaz A, Tirado-Sánchez A, Paredes-Farrera F, Moreno-Moreno J, Araiza J, González GM. Oral involvement in mucormycosis. A retrospective study of 55 cases. Enferm Infecc Microbiol Clin (Engl Ed) 2020;S0213-005X(Suppl 20):30293-7. Doi: 10.1016/j.eimc. 2020.09.003

4 Rai S, Misra D, Misra A, Jain A, Jain P, Dhawan A. Palatal mucormycosis masquerading as bacterial and fungal osteomyelitis: a rare case report. Contemp Clin Dent 2018;9(02): 309-313

5 Cohen A, Shoukair FL, Korem M, Shaulov A, Casap N. Successful mandibular mucormycosis treatment in the severely neutropenic patient. J Oral Maxillofac Surg 2019;77(06):1209. e1-1209.e12

6 Pandilwar PK, Khan K, Shah K, Sanap M, K S AU, Nerurkar S. Mucormycosis: A rare entity with rising clinical presentation in immunocompromised hosts. Int J Surg Case Rep 2020;77:57-61

7 De Leonardis F, Perillo T, Giudice G, Favia G, Santoro N. Recurrent rhino-ocular-cerebral mucormycosis in a leukemic child: a case report and review of pediatric literature. Pediatr Rep 2015;7(03):5938

8 Srivastava N, Bansal V, Kantoor P. Palatal mucormycosis in an infant. J Dent Child (Chic) 2015;82(03):153-156

9 Samanta DR, Senapati SN, Sharma PK, Shruthi BS, Paty PB, Sarangi G. Hard palate perforation in acute lymphoblastic leukemia due to mucormycosis - a case report. Indian J Hematol Blood Transfus 2009;25(01):36-39

10 Kalaskar RR, Kalaskar AR, Ganvir S. Oral mucormycosis in an 18month-old child: a rare case report with a literature review. J Korean Assoc Oral Maxillofac Surg 2016;42(02):105-110
11 V Ramesh DNS, Anjum G, Rukmangada T, Patil N. Rhinocerebral maxillary mucormycosis: a palatal ulcer. Indian J Dent Res 2020; 31(04):652-655

12 De oliveira CC, Ferreira AEC, Verde MEQL, et al. Extensive palatal mucormycosis in an elderly patient: case report. Oral Surgery, Oral Medicine, Oral Pathology and Oral Radiology 2020;129(1): e109https://doi.org/10.1016/j.00oo.2019.06.465

13 Barrak HA. Hard palate perforation due to mucormycosis: report of four cases. J Laryngol Otol 2007;121(11):1099-1102

14 Piccin A, Russell JD, Fleming P, et al. Invasive rhino-maxillary mucormycosis diagnosed before HSCT. Pediatr Blood Cancer 2008;50(02):393-395

15 Abuali MM, Posada R, Del Toro G, et al. Rhizomucor variabilis var. regularior and Hormographiella aspergillata infections in a leukemic bone marrow transplant recipient with refractory neutropenia. J Clin Microbiol 2009;47(12):4176-4179

16 Al Akhrass F, Debiane L, Abdallah L, et al. Palatal mucormycosis in patients with hematologic malignancy and stem cell transplantation. Med Mycol 2011;49(04):400-405

17 France K, Stoopler ET, Tanaka TI. Palatal swelling in a patient with refractory leukemia. JAMA Dermatol 2019;155(01):109-110

18 Madney Y, Khedr R, Ahmed N, et al. Overview and outcome of mucormycosis among children with cancer: report from the Children's Cancer Hospital Egypt. Mycoses 2019;62(11):984-989

19 Vučicévić Boras V, Jurlina M, Brailo V, et al. Oral mucormycosis and aspergillosis in the patient with acute leukemia. Acta Stomatol Croat 2019;53(03):274-277

20 Doni BR, Peerapur BV, Thotappa LH, Hippargi SB. Sequence of oral manifestations in rhino-maxillary mucormycosis. Indian J Dent Res 2011;22(02):331-335

21 Manjunatha BS, Das N, Sutariya RV, Ahmed T. Mucormycosis of the hard palate masquerading as carcinoma. Clin Pract 2012;2 (01):e28

22 Mathur VP, Dhillon JK, Kalra G. Oral health in children with leukemia. Indian J Palliat Care 2012;18(01):12-18

23 Cornely OA, Alastruey-Izquierdo A, Arenz D, et al; Mucormycosis ECMM MSG Global Guideline Writing Group. Global guideline for the diagnosis and management of mucormycosis: an initiative of the European Confederation of Medical Mycology in cooperation with the Mycoses Study Group Education and Research Consortium. Lancet Infect Dis 2019;19(12):e405-e421

24 Jeong W, Keighley C, Wolfe R, et al. The epidemiology and clinical manifestations of mucormycosis: a systematic review and metaanalysis of case reports. Clin Microbiol Infect 2019;25(01):26-34

25 Lanternier F, Dannaoui E, Morizot G, et al; French Mycosis Study Group. A global analysis of mucormycosis in France: the RetroZygo Study (2005-2007). Clin Infect Dis 2012;54(Suppl 1):S35-S43

26 Marty FM, Cosimi LA, Baden LR. Breakthrough zygomycosis after voriconazole treatment in recipients of hematopoietic stem-cell transplants. N Engl J Med 2004;350(09):950-952

27 Kontoyiannis DP, Lionakis MS, Lewis RE, et al. Zygomycosis in a tertiary-care cancer center in the era of Aspergillus-active antifungal therapy: a case-control observational study of 27 recent cases. J Infect Dis 2005;191(08):1350-1360

28 Kauffman CA. Zygomycosis: reemergence of an old pathogen. Clin Infect Dis 2004;39(04):588-590

29 Pagano L, Gleissner B, Fianchi L. Breakthrough zygomycosis and voriconazole. J Infect Dis 2005;192(08):1496-1497, author reply 1497

30 Abidi MZ, Sohail MR, Cummins N, et al. Stability in the cumulative incidence, severity and mortality of 101 cases of invasive mucormycosis in high-risk patients from 1995 to 2011: a comparison of eras immediately before and after the availability of voriconazole and echinocandin-amphotericin combination therapies. Mycoses 2014;57(11):687-698 